\title{
Local thermal observables in spatially open FRW spaces
}

\author{
Slava Emelyanov* \\ Arnold Sommerfeld Center for Theoretical Physics, \\ Ludwig Maximilian University (LMU), \\ 80333 Munich, Germany
}

\begin{abstract}
Certain local thermal observables are considered in well-known examples of spatially open FRW spaces: Milne, open de Sitter and anti-de Sitter as well as Einstein static universes. Another value for fixing the ambiguity in defining the Wick square and, hence, the local temperature is motivated in the last example. Physical consequences of that choice are discussed for static and conformal vacua in those spaces.
\end{abstract}

Keywords: conformal field theory in curved spaces, local thermal observables, quantum vacua

*Electronic address: viacheslav.emelyanov@physik.uni-muenchen.de 


\section{INTRODUCTION}

In this paper I will explore, in particular, thermal characteristics of states considered in [1]. A framework employed below is based on the idea that one can construct microscopic quantities from the field products and its derivatives which are sensitive to thermal properties

of a certain quantum state [2, 3]. Among of these local thermal observables are a local temperature and a thermal energy-momentum tensor. Thus, computing them in a quantum state under consideration and in a some reference thermal one, one may decide to which extent it is legitimate to ascribe macroscopic thermal observables to it as well as whether it corresponds to a local thermal equilibrium.

Although there exists in general no global thermal states in curved spacetimes to be taken as reference ones, it is not the case in the problem under scrutiny. Specifically, I shall consider observers moving along geodesics corresponding to the integral curves of the conformal Killing vector field in spatially open FRW spaces among of which are Milne, open de Sitter (dS), anti-de Sitter (AdS) spaces as well as Einstein static universe (ESU). ${ }^{1}$ Therefore, a conformal Kubo-Martin-Schwinger (KMS) state [4] is chosen in all of these cases (except the last one, wherein it is just a KMS state) as the reference thermal one.

In Section III, I consider the contracting and expanding Milne universes. An alternative quantization being equivalent to the standard one and relation with the conformal vacuum are studied. Thermal properties of known states are discussed in the framework briefly outlined above. In Section III, I deal with AdS spacetime, wherein an alternative quantization of the conformal non-interacting scalar field on the AdS hyperboloid will be demonstrated that is unitary equivalent to the standard one [5] and the existence of which has been motivated in [1]. Along the line of Section II, a discussion of the thermal properties of the static and conformal vacua is presented. In Section IV, I discuss thermal local observables in the cases of open dS space as well as open Einstein static universe and compare them with those for closed dS space and the closed ESU. In Section V I provide final concluding remarks.

The sign convention for the metric tensor as well as the definition of the Riemann tensor are the same as in [1]. The fundamental constants are set to unity throughout this paper.

\section{MILNE SPACETIME}

The Milne universe is a subspace of Minkowski spacetime lying in the future lightcone originating from a given point $\mathrm{O}$ of the manifold. Its line element has the form of the open FRW universe with the scale factor exponentially growing with the conformal time, i.e.

$$
d s^{2}=a^{2}(\bar{\eta})\left(d \bar{\eta}^{2}-d \bar{\chi}^{2}-\sinh ^{2} \bar{\chi} d \bar{\Omega}^{2}\right)
$$

\footnotetext{
${ }^{1}$ Spacetime is open or closed if the spatial section is $\mathbf{H}^{3}$ or $\mathbf{S}^{3}$, respectively.
} 
where $a(\bar{\eta})=e^{\bar{\eta}}$ is the scale factor and $d \bar{\Omega}^{2}=d \bar{\theta}^{2}+\sin ^{2} \bar{\theta} d \bar{\varphi}^{2}$ element of solid angle. By the same metric with a reversed direction of $\bar{\eta}$ one can cover the past lightcone of the origin O, wherein now $a(\bar{\eta})$ approaches zero for $\bar{\eta} \rightarrow+\infty$. The future and past lightcones will be called the upper and lower Milne universes in the following, respectively.

\section{A. Minkowski modes in Milne spacetime}

To simplify calculations of the Wick square (see below) in the conformal KMS state, one has to expand the quantum field through modes which when rescaled becomes positive frequency ones with respect to the conformal Killing vector $\partial_{\bar{\eta}}$ being the dilatation [6]. These modes have been found in [7] for the upper Milne wedge which specify the Minkowski vacuum. One can also obtain the minkowskian modes in the lower wedge by analytically continuing them from the Rindler spacetime to that wedge. Thus, one has

$$
\Phi_{p l m}\left(\bar{x}_{ \pm}\right)= \pm i^{l} e^{ \pm \frac{\pi p}{2}} \frac{e^{\mp i p \bar{\eta}_{ \pm}}}{a\left(\bar{\eta}_{ \pm}\right)} \frac{\Gamma(1+l+i p)}{\left(4 \pi \sinh \bar{\chi}_{ \pm}\right)^{\frac{1}{2}}} P_{i p-\frac{1}{2}}^{-l-\frac{1}{2}}\left(\cosh \bar{\chi}_{ \pm}\right) Y_{l m}(\bar{\Omega}), \quad p \in \mathbf{R}
$$

where $\eta_{ \pm}$and $\chi_{ \pm}$are defined through $x^{0}= \pm e^{ \pm \bar{\eta}_{ \pm}} \cosh \bar{\chi}_{ \pm}$and $|\mathbf{x}|=e^{ \pm \bar{\eta}_{ \pm}} \sinh \bar{\chi}_{ \pm}$, where the plus and minus indices refer to the upper and lower Milne wedges, respectively.

By using Eq. (2), one can define conformal modes specifying the conformal Milne vacua in the wedges as follows

$$
\begin{aligned}
& \Phi_{\omega l m}^{+}(x)=\alpha_{\omega l m} \Phi_{+\omega l m}(x)+\beta_{\omega l m} \Phi_{-\omega l m}^{*}(x), \\
& \Phi_{\omega l m}^{-}(x)=\alpha_{\omega l m} \Phi_{-\omega l m}^{*}(x)+\beta_{\omega l m} \Phi_{+\omega l m}(x),
\end{aligned}
$$

where $\omega \in \mathbf{R}^{+}$and the Bogolyubov coefficients are

$$
\alpha_{\omega l m}=\frac{\exp \left(+\frac{\pi \omega}{2}\right)}{(2 \sinh (\pi \omega))^{\frac{1}{2}}}, \quad \beta_{\omega l m}=-(-1)^{l} \frac{\exp \left(-\frac{\pi \omega}{2}\right)}{(2 \sinh (\pi \omega))^{\frac{1}{2}}} .
$$

Note that the modes $\Phi_{\omega l m}^{+}(x)$ vanish in the lower wedge, while $\Phi_{\omega l m}^{-}(x)$ are strictly zero in the upper Milne wedge and both of them can be analytically continued into Rindler space.

One can express the Minkowski modes $\Phi_{p l m}(x)$ through the upper and lower conformal Milne ones, i.e. $\Phi_{\omega l m}^{ \pm}(x)$, by exploiting Eq. (3) . In other words, the Minkowski vacuum can be represented as an excited state under $\left|0^{+}\right\rangle \otimes\left|0^{-}\right\rangle$, where $\left|0^{ \pm}\right\rangle$is the conformal Milne vacua in the upper and lower wedges, respectively. The crucial ingredient of the model that allows such representation is a presence of the conformal symmetry, because then the commutator $\left[\hat{\Phi}\left(x_{1}\right), \hat{\Phi}\left(x_{2}\right)\right]$ vanishes whenever $x_{1}$ and $x_{2}$ lie in the different Milne wedges as it has been noted in [8]. Thus, the field degrees of freedom in one wedge are mutually independent from those lying in the other wedge. 


\section{B. Local thermal observables}

It is argued in [2, 3] that a real thermometer is modeled in the theoretical language with the help of the Wick square of the field. This construction and a concept of the local equilibrium have been further studied in curved spacetimes [9, 10] (see also [11]). Specifically, the squared value of the local temperature $\mathrm{T}(x)$ measured by the thermometer in a given state $\omega^{2}$ is probed by the Wick square as follows

$$
\mathrm{T}^{2}(x)=12 \omega\left(: \hat{\Phi}^{2}(x):\right),
$$

wherein the Wick square is defined as : $\hat{\Phi}\left(x_{1}\right) \hat{\Phi}\left(x_{2}\right)$ : $\equiv \hat{\Phi}\left(x_{1}\right) \hat{\Phi}\left(x_{2}\right)-H\left(x_{1}, x_{2}\right) \hat{1}$, where $H\left(x_{1}, x_{2}\right)$ is the Hadamard parametrix canceling the divergent terms in the two-point function in the coincidence limit $x_{2} \rightarrow x_{1}$ [13].

The conformal KMS state $\omega_{\beta}$ specified by the KMS parameter $\beta$ (the inverse temperature) can be chosen as a thermal reference one. The two-point function in $\omega_{\beta}$ equals

$$
\omega_{\beta}\left(\hat{\Phi}\left(\bar{x}_{1}\right) \hat{\Phi}\left(\bar{x}_{2}\right)\right)=\frac{i}{2 \pi a\left(\bar{\eta}_{1}\right) a\left(\bar{\eta}_{2}\right)} \int_{-\infty}^{+\infty} d k \frac{1}{1-e^{-\beta k}} \int_{-\infty}^{+\infty} d \bar{\eta} \Delta_{\mathrm{r}}\left(\bar{\eta}+\bar{\eta}_{1} ; \bar{\eta}_{2}\right) e^{i k \bar{\eta}},
$$

where $\Delta_{\mathrm{r}}\left(\bar{x}_{1}, \bar{x}_{2}\right)$ is the rescaled casual propagator, i.e. $\left[\hat{\Phi}_{\mathrm{r}}\left(\bar{x}_{1}\right), \hat{\Phi}_{\mathrm{r}}\left(\bar{x}_{2}\right)\right]=i \Delta_{\mathrm{r}}\left(\bar{x}_{1}, \bar{x}_{2}\right) \hat{1}$ and $\hat{\Phi}_{\mathrm{r}}(\bar{x}) \equiv a(\bar{\eta}) \hat{\Phi}(\bar{x})$. Exploiting the previous Subsection, one finds

$$
\mathrm{T}^{2}(\bar{x})=\frac{1}{a^{2}(\bar{\eta})}\left(\frac{1}{\beta^{2}}-\frac{1}{4 \pi^{2}}\right) .
$$

The Hadamard parametrix in this model coincides with the Minkowski two-point function, therefore, the local temperature in the Minkowski vacuum state is strictly zero. However, the Wick square in the conformal Milne vacuum is negative and coincides with the right-hand side of Eq. (7) in the limit $\beta \rightarrow+\infty$, s.t. $\mathrm{T}^{2}(\bar{x})=-1 / 4 \pi^{2} a^{2}(\bar{\eta})$.

The renormalized vacuum expectation value of the energy-momentum tensor $\hat{T}_{\nu}^{\mu}(x)$ vanishes in the Minkowski vacuum. Hence, one obtains

$$
\omega_{\beta}\left(\hat{T}_{\nu}^{\mu}(\bar{x})\right)=\frac{1}{480 \pi^{2} a^{4}(\bar{\eta})}\left(\left(\frac{2 \pi}{\beta}\right)^{4}-1\right)\left(\delta_{\nu}^{\mu}-\frac{4}{3} \delta_{i}^{\mu} \delta_{i \nu}\right),
$$

wherein $i$ runs from 1 to 3 . The thermal energy-momentum tensor $E_{\nu}^{\mu}(\bar{x})$ in this framework is, however, only a part of the total energy-momentum tensor $T_{\nu}^{\mu}(\bar{x})$ (denoted by $\epsilon_{\nu}^{\mu}$ in [10]), such that it equals

$$
\omega_{\beta}\left(\hat{E}_{\nu}^{\mu}(\bar{x})\right)=\frac{1}{120 \pi^{2} a^{4}(\bar{\eta})}\left(1+\frac{4 \pi^{4}}{\beta^{4}}-\frac{5 \pi^{2}}{\beta^{2}}\right)\left(\delta_{\nu}^{\mu}-\frac{4}{3} \delta_{i}^{\mu} \delta_{i \nu}\right) .
$$

\footnotetext{
${ }^{2}$ It is a standard designation of a state in the algebraic approach to the quantum field theory, see [12, 13]. I use it below for the convenience.
} 
Both $\omega_{\beta}\left(\hat{T}_{\nu}^{\mu}(\bar{x})\right)$ and $\omega_{\beta}\left(\hat{E}_{\nu}^{\mu}(\bar{x})\right)$ are traceless and vanish in the Minkowski vacuum, but $\omega_{\beta}\left(\hat{T}_{0}^{0}(\bar{x})\right)$ is less then zero in the conformal Milne vacuum, while $\omega_{\beta}\left(\hat{E}_{0}^{0}(\bar{x})\right)$ is positive in it. Note that neither $\omega_{\beta}\left(\hat{T}_{\nu}^{\mu}(\bar{x})\right)$ nor $\omega_{\beta}\left(\hat{E}_{\nu}^{\mu}(\bar{x})\right)$ are proportional to $\mathrm{T}^{4}(\bar{x})$ as this the case for the pure thermal radiation.

\section{OPEN ANTI-DE SITTER SPACETIME}

Anti-de Sitter spacetime can be imagined as a four dimensional hyperboloid embedded in a five-dimensional space $\mathbf{R}^{5}$ with the line element $d s^{2}=\eta_{a b} d x^{a} d x^{b}$, where $a$ and $b$ run from 0 to 4 and $\eta_{a b}=\operatorname{diag}(+,-,-,-,+)$, i.e. $\eta_{a b} x^{a} x^{b}=1$. This spacetime is pathological from the point of view that it is not globally hyperbolic. The first reason lies in that its topology is $\mathbf{S} \times \mathbf{R}^{3}$, so that it possesses closed time-like curves. This feature is cured by unwrapping the circle $\mathbf{S}$ and considering instead its universal covering $\mathbf{R}$. The second reason consists in that its spatial infinity is time-like. Therefore, one has to set a boundary condition there to fix the energy flux through it. These allow to have a well-defined quantum theory in the AdS hyperboloid [5].

Among of possible parameterizations of the AdS hyperboloid, I shall consider so-called open coordinates. The line element in these coordinates becomes

$$
d s^{2}=a^{2}(\bar{\eta})\left(d \bar{\eta}^{2}-d \bar{\chi}^{2}-\sinh ^{2} \bar{\chi} d \bar{\Omega}^{2}\right),
$$

where $a(\bar{\eta})=1 / \cosh \bar{\eta}$. By the same metric one can describe geometry inside the wedges for which $\eta \in(2 \pi k, \pi+2 \pi k)$, where $\eta$ is the time coordinate in the static frame [1] and $k \in \mathbf{Z}$. The geodesics of AdS space correspond to comoving geodesics in open AdS space, i.e. integral curves of the conformal Killing vector $\xi=\partial_{\bar{\eta}}$, i.e. the dilatation [6].

For the analysis below it is needed to introduce coordinates that cover the rest part of the AdS hyperboloid. They can be obtained by setting $\bar{\eta}=\tilde{\chi}-\frac{i \pi}{2}$ and $\bar{\chi}=\tilde{\eta}+\frac{i \pi}{2}$. These coordinates correspond to a parametrization of the wedges with $\eta \in\left(-\frac{\pi}{2}+2 \pi k,+\frac{\pi}{2}+2 \pi k\right)$, such that the line element equals

$$
d s^{2}=a^{2}(\tilde{\chi})\left(d \tilde{\eta}^{2}-d \tilde{\chi}^{2}-\cosh ^{2} \tilde{\eta} d \tilde{\Omega}^{2}\right),
$$

where $a(\tilde{\chi})=1 / \sinh \tilde{\chi}$. Up to the scale factor it is similar to the Rindler universe in the spherical coordinates [7]. Therefore, the line element (11) is conformally related with the Minkwoski one, specifically

$$
d s^{2}=\frac{4 \eta_{\mu \nu} d x^{\mu} d x^{\nu}}{\left(1+\eta_{\lambda \rho} x^{\lambda} x^{\rho}\right)^{2}},
$$

where $x^{\mu}=(r \tanh \tilde{\eta}, r \sin \theta \cos \varphi, r \sin \theta \sin \varphi, r \cos \theta)$ and $r=e^{\tilde{x}} \cosh \tilde{\eta}$, such that $t^{2}-r^{2}<0$. If one takes $r^{2}-t^{2}>0$, then this metric can be transformed to the form it has in Eq. (10). Thus, one can cover the AdS hyperboloid by domains being conformally related to Minkowski 
space. Note that changing the sign inside the scale factor in (12), one obtains the de Sitter line element.

The Killing vector

$$
\zeta=x^{3} \partial_{x^{0}}+x^{0} \partial_{x^{3}}=\cos \tilde{\theta} \partial_{\tilde{\eta}}-\tanh \tilde{\eta} \sin \tilde{\theta} \partial_{\tilde{\theta}}
$$

in those wedges is timelike for $\tilde{\theta} \in\{0, \pi\}^{3}$ and sets dynamics for an observer moving with a constant four-acceleration through AdS space, while $\sigma=\partial_{x^{0}}$ is the conformal Killing vector. ${ }^{4}$ Due to the conformal symmetry, one can expand the rescaled field through the plane modes or through the boost ones, i.e. modes being eigenfunctions of $\sigma$ or the boost operator $\zeta$ in Minkowski space, respectively. On the other hand, an observer moving along integral curves of $\zeta$ in AdS space defines Unruh modes up to the conformal factor. Hence, these vacua

are thermally related as it has been first found in [14] by employing the Unruh-DeWitt detector [15].

\section{A. Static AdS modes in open AdS space}

As in the case of the Milne universe it is convenient to expand the field through the modes which when rescaled are eigenfunctions of $\xi$ and still define the static vacuum [5]. I argued in [1] that these modes must exist. I show the modes below in this Subsection.

For the wedge $\eta \in\left(-\frac{\pi}{2},+\frac{\pi}{2}\right)$ these modes are

$$
\Phi_{p l m}(\tilde{x})=-i^{l+1} \frac{e^{-i p \tilde{\chi}}}{a(\tilde{\chi})} \frac{\Gamma(1+l+i p)}{(4 \pi i \cosh \tilde{\eta})^{\frac{1}{2}}} P_{i p-\frac{1}{2}}^{-l-\frac{1}{2}}(i \sinh \tilde{\eta}) Y_{l m}(\tilde{\Omega}), \quad p \in \mathbf{R}
$$

which are normalized on the effective Cauchy surface $\Sigma=\Sigma_{1} \cup \Sigma_{2}$, i.e. these modes correspond to the "transparent" boundary conditions [5].

Performing an analytic continuation into the wedges $\eta \in(0,+\pi)$ and $\eta \in(-\pi, 0)$ according to $\bar{\eta}_{+}=\tilde{\chi}-\frac{i \pi}{2}, \bar{\chi}_{+}=\tilde{\eta}+\frac{i \pi}{2}$ and $\bar{\eta}_{-}=-\tilde{\chi}-\frac{i \pi}{2}, \bar{\chi}_{-}=\tilde{\eta}+\frac{i \pi}{2}$, respectively, one obtains

$$
\Phi_{p l m}\left(\bar{x}_{ \pm}\right)= \pm i^{l} e^{ \pm \frac{\pi p}{2}} \frac{e^{\mp p \bar{\eta}_{ \pm}}}{a\left(\bar{\eta}_{ \pm}\right)} \frac{\Gamma(1+l+i p)}{\left(4 \pi \sinh \bar{\chi}_{ \pm}\right)^{\frac{1}{2}}} P_{i p-\frac{1}{2}}^{-l-\frac{1}{2}}\left(\cosh \bar{\chi}_{ \pm}\right) Y_{l m}\left(\bar{\Omega}_{ \pm}\right) .
$$

Having these modes, it is straightforward to obtain the relation between the conformal and static vacua in AdS space [1]. Indeed, the conformal modes are

$$
\begin{aligned}
& \Phi_{\omega l m}^{+}(x)=\alpha_{\omega l m} \Phi_{+\omega l m}(x)+\beta_{\omega l m} \Phi_{-\omega l m}^{*}(x), \\
& \Phi_{\omega l m}^{-}(x)=\alpha_{\omega l m} \Phi_{-\omega l m}^{*}(x)+\beta_{\omega l m} \Phi_{+\omega l m}(x),
\end{aligned}
$$

\footnotetext{
${ }^{3}$ It is not a restriction, because the rotation group $\mathrm{SO}(3)$ is a subgroup of the AdS symmetry group $\mathrm{SO}(2,3)$, so that one can always set those values of $\tilde{\theta}$ without loss of generality.

4 See [6] for more details.
} 
where $\omega \in \mathbf{R}^{+}$and

$$
\alpha_{\omega l m}=\frac{\exp \left(+\frac{\pi \omega}{2}\right)}{(2 \sinh (\pi \omega))^{\frac{1}{2}}}, \quad \beta_{\omega l m}=-(-1)^{l} \frac{\exp \left(-\frac{\pi \omega}{2}\right)}{(2 \sinh (\pi \omega))^{\frac{1}{2}}} .
$$

One can show that the modes $\Phi_{\omega l m}^{+}(x)$ vanish in the wedges where $\eta \in(-\pi+2 \pi k, 2 \pi k)$, while the modes $\Phi_{\omega l m}^{-}(x)$ vanish in the wedges where $\eta \in(2 \pi k,+\pi+2 \pi k), k \in \mathbf{Z}$.

The modes given in Eq. (14) or Eq. (15) define the static vacuum. Indeed, the two-point function is

$$
\omega_{\mathrm{S}}\left(\hat{\Phi}\left(x_{1}\right) \hat{\Phi}\left(x_{2}\right)\right)=\sum_{l m} \int_{-\infty}^{+\infty} d p \Phi_{p l m}^{\mathrm{S}}\left(x_{1}\right) \Phi_{p l m}^{\mathrm{S} *}\left(x_{2}\right)=\frac{1}{8 \pi^{2}} \frac{\cos \chi_{1} \cos \chi_{2}}{\cos (\Delta \eta-i \varepsilon)-\cos (\zeta)}
$$

where it has been already rewritten in the static AdS coordinates, $\cos \zeta=\cos \left(\chi_{1}-\chi_{2}\right)+$ $\sin \chi_{1} \sin \chi_{2}(\cos \Theta-1)$ and $\cos \Theta=\cos \theta_{1} \cos \theta_{2}+\sin \theta_{1} \sin \theta_{2} \cos \left(\phi_{1}-\phi_{2}\right)$. Comparing it with the two-point function for the closed Einstein static universe derived in [15] conformally mapped to AdS space, one concludes the modes given in Eq. (15) are unitary equivalent to the static ones for the "transparent" boundary conditions [5].

\section{B. Local thermal observables}

In analogous manner to Subsec. IIB, one finds the squared value of the local temperature ascribed to the conformal KMS state with the inverse temperature $\beta$ :

$$
\mathrm{T}^{2}(\bar{x})=\frac{1}{a^{2}(\bar{\eta})}\left(\frac{1}{\beta^{2}}-\frac{1}{4 \pi^{2}}\right)+\frac{R}{24 \pi^{2}}-12 \alpha_{0} R,
$$

where $\alpha_{0}$ is due to ambiguity in defining the Wick square [16] and $R=-6\left(a^{\prime \prime} / a-1\right) / a^{2}$ Ricci scalar equaling to +12 in AdS space. If one sets $\alpha_{0}=1 / 288 \pi^{2}$, then the Wick square is a conformally invariant field [17] (see also [11]). Another motivation for this choice of $\alpha_{0}$ is given in [10, 11]. This value of $\alpha_{0}$ is taken for granted in this Subsection.

The AdS vacuum restricted to open AdS space is a conformal KMS state with $\beta=2 \pi$. The squared local temperature of the conformal vacuum is negative and equals $-1 / 4 \pi^{2} a^{2}(\bar{\eta})$.

Taking into account the renormalized vacuum expectation value of $\hat{T}_{\nu}^{\mu}(\bar{x})$ in the AdS vacuum in open AdS space, one derives

$$
\omega_{\beta}\left(\hat{T}_{\nu}^{\mu}(\bar{x})\right)=\frac{1}{960 \pi^{2}} \delta_{\nu}^{\mu}+\frac{1}{480 \pi^{2} a^{4}(\bar{\eta})}\left(\left(\frac{2 \pi}{\beta}\right)^{4}-1\right)\left(\delta_{\nu}^{\mu}-\frac{4}{3} \delta_{i}^{\mu} \delta_{i \nu}\right),
$$

whereas for the thermal energy-momentum tensor $\hat{E}_{\nu}^{\mu}(\bar{x})$ one finds an expression to be structurally rather different from the right-hand side of Eq. (19).

\footnotetext{
${ }^{5}$ Note that the "reflective" boundary conditions [5] are realized by taking the modes $\mathbf{P}_{ \pm} \Phi_{p l m}(x)$, where $\mathbf{P}_{ \pm}=\frac{1}{2}\left(\mathbf{1} \pm \mathfrak{I}_{\mathrm{C}}\right)$ and $\mathfrak{I}_{\mathrm{C}}$ is the so-called conformal inversion $[6]$.
} 


\section{DISCUSSION}

An analogous result to (18) one obtains for the local temperature squared in open de Sitter spacetime, where $R=-12$ and $a(\bar{\eta})=1 / \sinh \bar{\eta}$ in that equation. The total renormalized energy-momentum tensor $\hat{T}_{\nu}^{\mu}(\bar{x})$ in the conformal KMS state $\omega_{\beta}$ is functionally given by the same Eq. (19). The conformal KMS state with $\beta=2 \pi$ corresponds to the conformal or Chernikov-Tagirov vacuum [15] defined on the whole dS hyperboloid.

As has been mentioned above, the Wick square is ambiguous [16]. This means one has to impose an extra condition to get rid of that. The value of $\alpha_{0}=1 / 288 \pi^{2}$ has been motivated in [10, 11]. However, one has to set a zero value of $\alpha_{0}$ to have a zero local temperature for an observer freely moving along the time translation Killing vector in the open Einstein static universe. Indeed, the local temperature squared can be immediately obtained from Eq. (18) by setting $a(\bar{\eta})=1$, so that if the quantum field is in the static ESU vacuum, then $\beta \rightarrow+\infty$. Hence, $\mathrm{T}^{2}(\bar{x})=0$ if and only if $\alpha_{0}=0$, where $R=+6$ in the open Einstein static universe has been taken into account. The renormalized expectation values of the total and thermal energy-momentum tensors in the KMS state defined with respect to the Killing vector $\partial_{\bar{\eta}}$ coincide and are equal to

$$
\omega_{\beta}\left(\hat{T}_{\nu}^{\mu}(\bar{x})\right)=\omega_{\beta}\left(\hat{E}_{\nu}^{\mu}(\bar{x})\right)=\frac{\pi^{2}}{60 \beta^{4}}\left(\delta_{\nu}^{\mu}-\frac{4}{3} \delta_{i}^{\mu} \delta_{i \nu}\right) .
$$

Note that they are both proportional to $\mathrm{T}^{4}(\bar{x})$ for vanishing $\alpha_{0}$. This result then is similar to that in Minkowski space for an inertial observer, wherein, however, the value of $\alpha_{0}$ is irrelevant, because the scalar curvature vanishes.

As has been noted above, the Chernikov-Tagirov state in closed de Sitter space is also the conformal vacuum defined with respect to the conformal Killing vector $\partial_{\eta}$. The local temperature squared is

$$
\mathrm{T}^{2}(x)=\frac{6}{\pi^{2} a^{2}(\eta)} \sum_{n=0}^{+\infty} \frac{n}{e^{\beta n}-1}+\frac{R}{24 \pi^{2}}-12 \alpha_{0} R,
$$

where $a(\eta)=1 / \sin \eta$ and $R=-6\left(a^{\prime \prime} / a+1\right) / a^{2}$ Ricci scalar equaling to -12 in dS space. This result is a slight generalisation of that obtained in [18]. The expectation value of $\hat{T}_{\nu}^{\mu}(x)$ in the conformal KMS state defined with respect to $\partial_{\eta}$ is given by

$$
\omega_{\beta}\left(\hat{T}_{\nu}^{\mu}(x)\right)=\frac{1}{960 \pi^{2}} \delta_{\nu}^{\mu}+\frac{1}{2 \pi^{2} a^{4}(\eta)} \sum_{n=0}^{+\infty} \frac{n^{3}}{e^{\beta n}-1}\left(\delta_{\nu}^{\mu}-\frac{4}{3} \delta_{i}^{\mu} \delta_{i \nu}\right),
$$

where the renormalized vacuum expectation value of $\hat{T}_{\nu}^{\mu}(x)$ in the Chernikov-Tagirov state has been taken into account [15], while $\omega_{\beta}\left(\hat{E}_{\nu}^{\mu}(x)\right)$ has structurally a different form in comparison with $\omega_{\beta}\left(\hat{T}_{\nu}^{\mu}(x)\right)$ given in Eq. (22).

A comoving observer in closed dS space moving along curves with the tangent vector $\partial_{\eta}$ has to register in the standard interpretation a thermal bath with the Gibbons-Hawking 
temperature $\mathrm{T}_{\mathrm{GH}}=1 / 2 \pi[20]$. This implies $\alpha_{0}=1 / 192 \pi^{2}$. However, the local temperature is zero if $\alpha_{0}=1 / 288 \pi^{2}[10,11]$.

These energy-momentum tensors, i.e. $\hat{T}_{\nu}^{\mu}(x)$ and $\hat{E}_{\nu}^{\mu}(x)$, in the thermal state coincide in the closed Einstein static universe. The local temperature squared can be obtained from Eq. (21) by setting $a(\eta)=1$, such that $R=-6$ and $\alpha_{0}=1 / 288 \pi^{2}$ [1]. Employing the renormalized vacuum expectation value of $\hat{T}_{\nu}^{\mu}(x)$ for the closed ESU [15, 19], one obtains

$$
\omega_{\beta}\left(\hat{T}_{\nu}^{\mu}(x)\right)=\omega_{\beta}\left(\hat{E}_{\nu}^{\mu}(x)\right)=\frac{1}{2 \pi^{2}}\left(\sum_{n=0}^{+\infty} \frac{n^{3}}{e^{\beta n}-1}+\frac{1}{240}\right)\left(\delta_{\nu}^{\mu}-\frac{4}{3} \delta_{i}^{\mu} \delta_{i \nu}\right),
$$

which are clearly not proportional to the quartic value of the local temperature as in the case of the pure thermal radiation.

\section{CONCLUDING REMARKS}

In the present paper I have considered certain local thermal observables in the wellknown examples of spatially open FRW spaces for the conformal linear field theory. These observables have been put forward in a series of articles [2, 3, 9 [11] with a goal to define a local thermal equilibrium under the influence of external fields, in particular, in curved spacetimes.

I have found that the ambiguity in defining the Wick square parametrized by $\alpha_{0}$ [16] has no universal value, i.e. depends on a particular situation. It has to be zero in the open Einstein static universe, otherwise the physical meaning of the local temperature $\mathrm{T}(x)$ as defined in [2, 3] is lost.

Assuming that $\alpha_{0}$ has to be zero for all open FRW universes considered above, one is forced to conclude that the local temperature of the Minkowski vacuum vanishes, but is real for the AdS vacuum restricted to open AdS space and imaginary for the Chernikov-Tagirov state restricted to open dS space. Thus, the physical meaning of the local temperature as a model for a real thermometer becomes lost in the last case.

The local temperature for the conformal vacua in Milne and open dS spaces are imaginary, but is real only for a short interval of $\bar{\eta}$ close to $\bar{\eta}=0$ in open anti-de Sitter space. However, the backreaction of the field being in the conformal vacuum is infinite on horizons, so that these vacua cannot anyway be physically realized.

I have considered in [1] spacetime that approaches the open Einstein static universe at future- and past-time infinities and has a phase when space looks like open anti-de Sitter spacetime. During the AdS phase one might await that a comoving observer detects a thermal radiation with temperature $\mathrm{T}=(2 \pi a(\bar{\eta}))^{-1}$ which vanishes at $\bar{\eta} \rightarrow \pm \infty$ [1]. Qualitatively it behaves itself as the local temperature $\mathrm{T}(x)$ around $\bar{\eta}=0$ and in the limits $\bar{\eta} \rightarrow \pm \infty$. However, $\mathrm{T}^{2}(x)$ still has no physical sense, because it possesses negative values.

To sum it up, the concept of the local temperature as originally defined is at least questionable. Perhaps, its appropriately modified version could be identified with the readings 
of a real thermometer. This demands, however, further investigations.

\section{ACKNOWLEDGMENTS}

This research is supported by TRR 33 "The Dark Universe".

[1] S. Emelyanov, "Freely moving observer in (quasi) anti-de Sitter space," Phys. Rev. D90, 044039 (2014), arXiv:gr-qc/1309.3905.

[2] D. Buchholz, I. Ojima and H. Roos, "Thermodynamic properties of non-equilibrium states in quantum field theory," Annals of Physics 297, 219 (2002), arXiv:hep-ph/0105051.

[3] D. Buchholz, "On hot bangs and the arrow of time in relativistic quantum field theory," Commun. Math. Phys. 237, 271 (2003), arXiv:hep-th/0301115.

[4] R. Haag, Local quantum physics. Fields, Particles, Algebras, (Springer-Verlag, 1996).

[5] S.J. Avis, C.J. Isham and D. Storey, "Quantum field theory in anti-de Sitter space-time," Phys. Rev. D18, 3565 (1978).

[6] S. Emelyanov, "Non-unitarity or hidden observables?," arXiv:gr-qc/1410.6149.

[7] T. Tanaka and M. Sasaki, "Quantized gravitational waves in the Milne universe," Phys. Rev. D55, 6061 (1997), arXiv:gr-qc/9610060.

[8] S.J. Olson and T.C. Ralph, "Entanglement between the future and the past in the quantum vacuum," Phys. Rev. Lett. 106, 110404 (2011), arXiv:quant-ph/1003.0720.

[9] D. Buchholz and J. Schlemmer, "Local temperature in curved spacetime," Class. Quantum Grav. 24, F25 (2007), arXiv:gr-qc/0608133.

[10] C. Solveen, "Local thermal equilibrium and KMS states in curved spacetime," Class. Quantum Grav. 29, 245015 (2012), arXiv:gr-qc/1211.0431.

[11] C. Solveen, Local equilibrium states in quantum field theory in curved spacetime (PhD Thesis, University of Göttingen, 2012).

[12] S. Hollands and R.M. Wald, "Quantum fields in curved space-time," arXiv:gr-qc/1401.2026.

[13] R.M. Wald, Quantum field theory in curved spacetime and black hole thermodynamics (University of Chicago Press, Chicago, 1994).

[14] S. Deser and O. Levin, "Accelerated detectors and temperature in (anti-) de Sitter spaces," Class. Quantum Grav. 14, L163 (1997), arXiv:gr-qc/9706018.

[15] N.D. Birrell and P.C.W. Davies, Quantum fields in curved space (CUP, 1982).

[16] S. Hollands and R.M. Wald, "Local Wick polynomials and time ordered products of quantum fields in curved spacetime," Commun. Math. Phys. 223, 289 (2001), arXiv:gr-qc/0103074.

[17] N. Pinamonti, "Conformal generally covariant quantum field theory: the scalar field and its Wick products," Commun. Math. Phys. 288, 1117 (2009), arXiv:math-ph/0806.0803. 
[18] A. Stottmeister, Zur Thermodynamik gekrümmter Raumzeiten (Ms Thesis, University of Göttingen, 2009).

[19] P. Candelas and J.S. Dowker, "Field theories on conformally related spacetimes: Some global considerations," Phys. Rev. D 19, 2902 (1979).

[20] G.W. Gibbons and S.W. Hawking, "Cosmological event horizons, thermodynamics, and particle creation," Phys. Rev. D15, 2738 (1977). 